\title{
Fast two-bit operations in inductively coupled flux qubits
}

\author{
J. Q. You, ${ }^{1,2, *}$ Y. Nakamura, ${ }^{1,3,4, \dagger}$ and Franco Nori ${ }^{1,5, *}$ \\ ${ }^{1}$ Frontier Research System, The Institute of Physical and Chemical Research (RIKEN), Wako-shi 351-0198, Japan \\ ${ }^{2}$ Department of Physics and National Laboratory for Surface Physics, Fudan University, Shanghai 200433, China \\ ${ }^{3}$ NEC Fundamental and Environmental Research Laboratories, Tsukuba, Ibaraki 305-8051, Japan \\ ${ }^{4}$ CREST, Japan Science and Technology Agency (JST), Kawaguchi, Saitama 332-0012, Japan \\ ${ }^{5}$ Center for Theoretical Physics, Physics Department, Center for the Study of Complex Systems, \\ University of Michigan, Ann Arbor, Michigan 48109-1120, USA
}

(Received 25 May 2004; published 31 January 2005)

\begin{abstract}
A central problem for implementing efficient quantum computing is how to realize fast operations (both oneand two-bit ones). However, this is difficult to achieve for a collection of qubits, especially for those separated far away, because the interbit coupling is usually much weaker than the intrabit coupling. Here we present an experimentally feasible method to effectively couple two flux qubits via a common inductance and treat both single and coupled flux qubits with more realistic models, which include the loop inductance. The main advantage of our proposal is that a strong interbit coupling can be achieved using a small inductance, so that two-bit as fast as one-bit operations can be easily realized. We also show the flux dependence of the transitions between states for the coupled flux qubits.
\end{abstract}

DOI: 10.1103/PhysRevB.71.024532

PACS number(s): 74.50.+r, 85.25.Cp, 03.67.Lx

\section{INTRODUCTION}

Josephson-junction circuits can exhibit quantum behaviors. Among qubits based on Josephson-junction circuits, the charge qubit realized in a Cooper-pair box can demonstrate quantum oscillations. ${ }^{1}$ An improved version of this circuit has showed quantum oscillations with a high quality factor. ${ }^{2}$ In addition to charge qubits, flux qubits achieved in a superconducting loop with one ${ }^{3}$ or three Josephson junctions ${ }^{4}$ have been studied and some of these have shown quantum dynamics. ${ }^{5}$ The phase qubit consists of a large-area currentbiased Josephson junction. ${ }^{6}$

Capacitive couplings of two superconducting qubits (both charge- ${ }^{7}$ and phase-types ${ }^{8}$ ) were attained recently in experiments, and quantum entanglement was observed in these systems. Also, controllable interbit couplings of charge qubits were proposed using a variable electrostatic transformer, ${ }^{9}$ a current-biased Josephson junction, ${ }^{10}$ and a tunable dc-SQUID. ${ }^{11}$ These interbit couplings can link nearestneighboring qubits. Actually, there are quantum-computing protocols (e.g., adiabatic quantum computing ${ }^{12}$ ) that only demand nearest-neighbor couplings. However, for more general quantum-computing protocols, it is desirable to achieve strong-enough couplings among nonneighboring qubits as well. When charge qubits are coupled by $L C$-oscillator modes ${ }^{13}$ or by an inductance,${ }^{14}$ long-range interbit couplings can be realized, but a very large value of the inductance is needed. An alternate way of coupling charge qubits was proposed using a Josephson junction. ${ }^{15-17}$ Moreover, the charge qubit can be very sensitive to the background charge fluctuations, which generate noise that severely limits the performance of charge-qubit devices and, unfortunately, is difficult to reduce.

In this paper, we present an experimentally feasible method to effectively couple two flux qubits. In contrast with the charge qubit, the flux qubit is insensitive to the charge noise. In this qubit, the major noise is due to the fluctuations of the magnetic fluxes. Estimations show that the flux qubit can have a relatively high quality factor. ${ }^{18}$ Here we include the effect of the loop inductance in a three-junction flux qubit and couple two flux qubits via a common inductance. Because the critical current of each Josephson junction in the flux qubit is larger than that in the charge qubit, we can produce a strong interbit coupling using an inductance as small as $20 \mathrm{pH}$ (corresponding to a loop diameter of approximately $16 \mu \mathrm{m}$ and comparable to the loop inductance of the single flux qubit currently achieved in experiments), and thereby two-bit as fast as one-bit operations can be easily achieved, improving the efficiency of quantum computing. Moreover, we show a different flux dependence of the state transitions in two coupled flux qubits. We find that, except for some specific values of the external flux, the forbidden transitions in the two coupled flux qubits become allowed when the parameters of the two qubits change from being initially equal to each other and then making these different.

Coupling two flux qubits by a mutual inductance was proposed in Refs. 19-21 and was recently realized in experiments. ${ }^{22,23}$ Here we treat both single and coupled flux qubits using more realistic models, which include the loop inductance. We numerically solve the Schrödinger equation to obtain the energy levels and the eigenstates of the fluxqubit systems. This numerical method allows us to extend our study to the larger inductance regime.

The paper is organized as follows. In Sec. II, we study a single flux qubit containing loop inductance. It is shown that the system can still be used to achieve a qubit even for a larger loop inductance of $L \sim 1 \mathrm{nH}$. Section III focuses on two flux qubits coupled by a common inductance. In Sec. IV, we study the state transitions induced by the microwave field. Section V deals with the circulating supercurrents and quantum measurement. Finally, the discussion and conclusion are given in Sec. VI. 

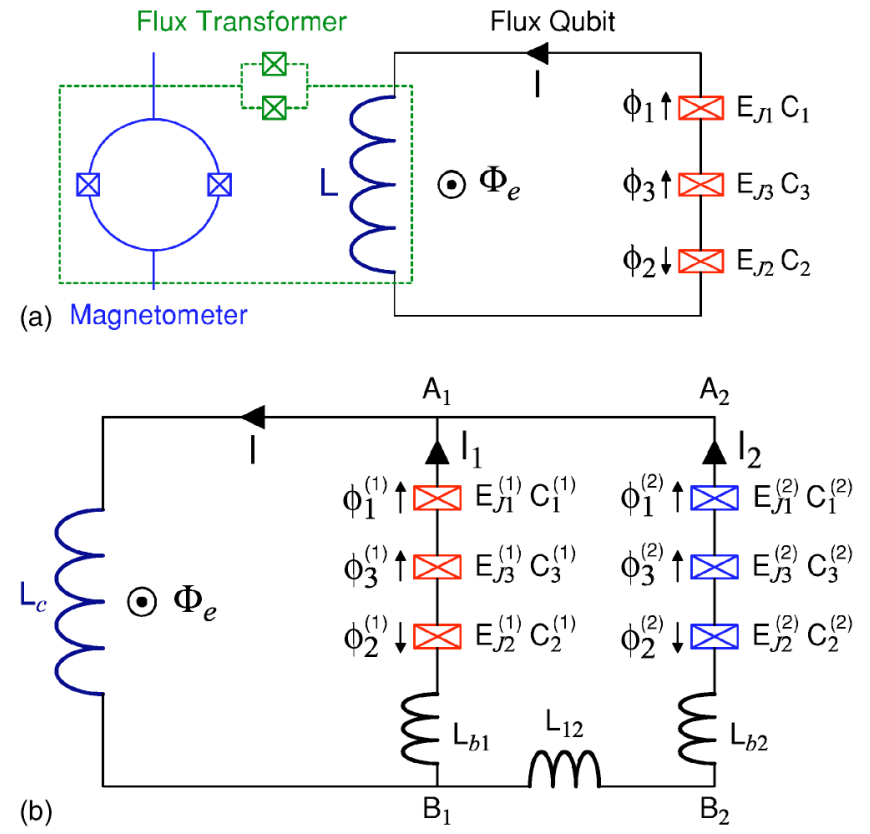

FIG. 1. (Color online) (a) A flux qubit, where an external magnetic flux $\Phi_{e}$ pierces the superconducting loop that contains three Josephson junctions and an inductance $L$. The Josephson energies and capacitances of the junctions are $E_{J 1}=E_{J 2}=E_{J}, C_{1}=C_{2}=C$, $E_{J 3}=\alpha E_{J}$, and $C_{3}=\alpha C$. Here we choose $\alpha=0.8$ and $E_{J}=35 E_{c}$, where $E_{c}=e^{2} / 2 C$. (b) Two flux qubits coupled by a common inductance $L_{c}$, where the external flux $\Phi_{e}$ is applied within the left loop $A_{1} L_{c} B_{1} A_{1}$. The parameters of each flux qubit are $E_{J 1}^{(i)}=E_{J 2}^{(i)}=E_{J}^{(i)}$, $C_{1}^{(i)}=C_{2}^{(i)}=C^{(i)}, E_{J 3}^{(i)}=\alpha_{i} E_{J}^{(i)}$, and $C_{3}^{(i)}=\alpha_{i} C^{(i)}$, with $i=1,2$. Here we choose $\alpha_{i}=0.8$ and $E_{J}^{(i)}=35 E_{c}^{(i)}$, where $E_{c}^{(i)}=e^{2} / 2 C^{(i)}$. To implement a readout of the flux-qubit states, a switchable superconducting flux transformer is employed to couple the dc-SQUID magnetometer with the inductance $L$ in (a) or $L_{c}$ in (b) during the quantum measurement. However, this coupling is switched off in the absence of a readout.

\section{SINGLE FLUX QUBIT}

\section{A. The model}

We first consider a single flux qubit in the absence of a quantum measurement, where the dc-SQUID magnetometer for measuring quantum states of the flux qubit is decoupled from the qubit. As shown in Fig. 1(a), the flux qubit consists of a superconducting loop with three Josephson junctions, and the total inductance of the whole loop is $L$. Fluxoid quantization around the loop imposes a constraint on the phase drops across the three junctions,

$$
\phi_{1}-\phi_{2}+\phi_{3}+2 \pi f^{\prime}=0
$$

where

$$
f^{\prime}=f+\frac{I L}{\Phi_{0}}
$$

Here, $\Phi_{0}=h / 2 e$ is the flux quantum,

$$
f=\Phi_{e} / \Phi_{0}
$$

represents the reduced magnetic flux, and

$$
I=I_{0} \sin \phi_{1},
$$

with $I_{0}=2 \pi E_{J} / \Phi_{0}$, is the circulating supercurrent.

When the loop inductance is included, the Hamiltonian of the single flux qubit is

$$
H=\frac{P_{p}^{2}}{2 M_{p}}+\frac{P_{m}^{2}}{2 M_{m}}+U\left(\phi_{p}, \phi_{m}\right),
$$

with the potential energy given by

$$
\begin{aligned}
U\left(\phi_{p}, \phi_{m}\right)= & E_{J}\left[2+\alpha-2 \cos \phi_{p} \cos \phi_{m}-\alpha \cos \left(2 \pi f^{\prime}\right.\right. \\
& \left.\left.+2 \phi_{m}\right)\right]+\frac{1}{2} L I^{2}
\end{aligned}
$$

Here

$$
\begin{gathered}
P_{k}=-i \hbar \frac{\partial}{\partial \phi_{k}}, k=p, m, \\
M_{p}=2 C\left(\Phi_{0} / 2 \pi\right)^{2}, \\
M_{m}=M_{p}(1+2 \alpha),
\end{gathered}
$$

and

$$
\begin{aligned}
& \phi_{p}=\frac{1}{2}\left(\phi_{1}+\phi_{2}\right), \\
& \phi_{m}=\frac{1}{2}\left(\phi_{1}-\phi_{2}\right) .
\end{aligned}
$$

Also, the supercurrent $I$ can be rewritten as

$$
I=I_{0} \sin \left(\phi_{p}+\phi_{m}\right) .
$$

The Hamiltonian (5) is reduced to Eq. (12) in Ref. 19 when $L \rightarrow 0$.

Figure 2 presents the contour plots of the periodic potential $U\left(\phi_{p}, \phi_{m}\right)$ for $f=0.5$ and $\alpha=0.8$. The numerical results show that the minima of the potential preserve the twodimensional centered cubic lattice even for a large loop inductance. For inductance ratio

$$
\beta_{L} \equiv L / L_{J}
$$

from zero to one (where $L_{J}=\Phi_{0} / 2 \pi I_{0}$ is the Josephsonjunction inductance), a well-defined double-well potential structure exists at each lattice point even though at higher energies the well shapes are modified by the loop inductance $L$. This double-well structure is required for achieving a twolevel system. As shown in Fig. 3, the lowest two levels of the single-qubit system are not significantly affected by the variation of $\beta_{L}$ (when $0 \leqslant \beta_{L} \lesssim 1$ ) because the corresponding two eigenstates are mainly contributed by the weakly $\beta_{L}$-dependent ground state in each well. However, since varying $L$ significantly modifies the well shapes at higher energies, the excited states within or above the wells (which, as seen in Fig. 3, dominantly contribute to the eigenstates corresponding to the third and higher levels) become pronouncedly $\beta_{L}$ dependent. Indeed, Fig. 3 shows that the top three levels are sensitive to the variation of $\beta_{L}$ (even when $0 \lesssim \beta_{L} \lesssim 1$ ). Moreover, with the loop inductance increasing to $\beta_{L} \approx 4$ [see Fig. 2(c)], a more distorted double-well structure appears at each lattice point, and a local energy minimum develops along the diagonal direction between every 


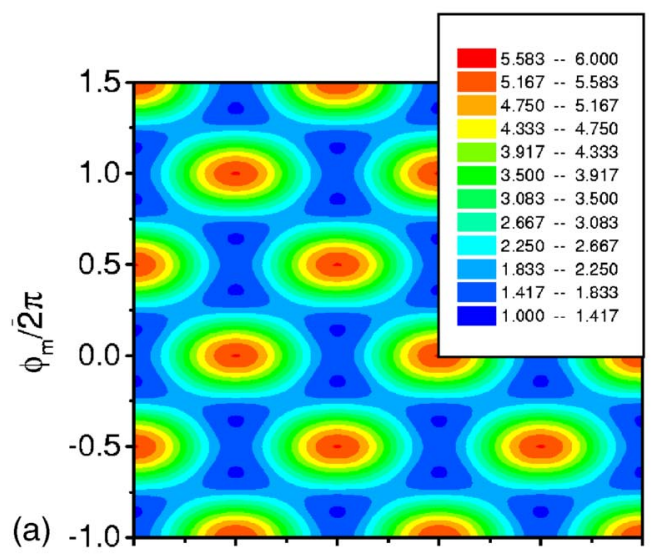

(b)
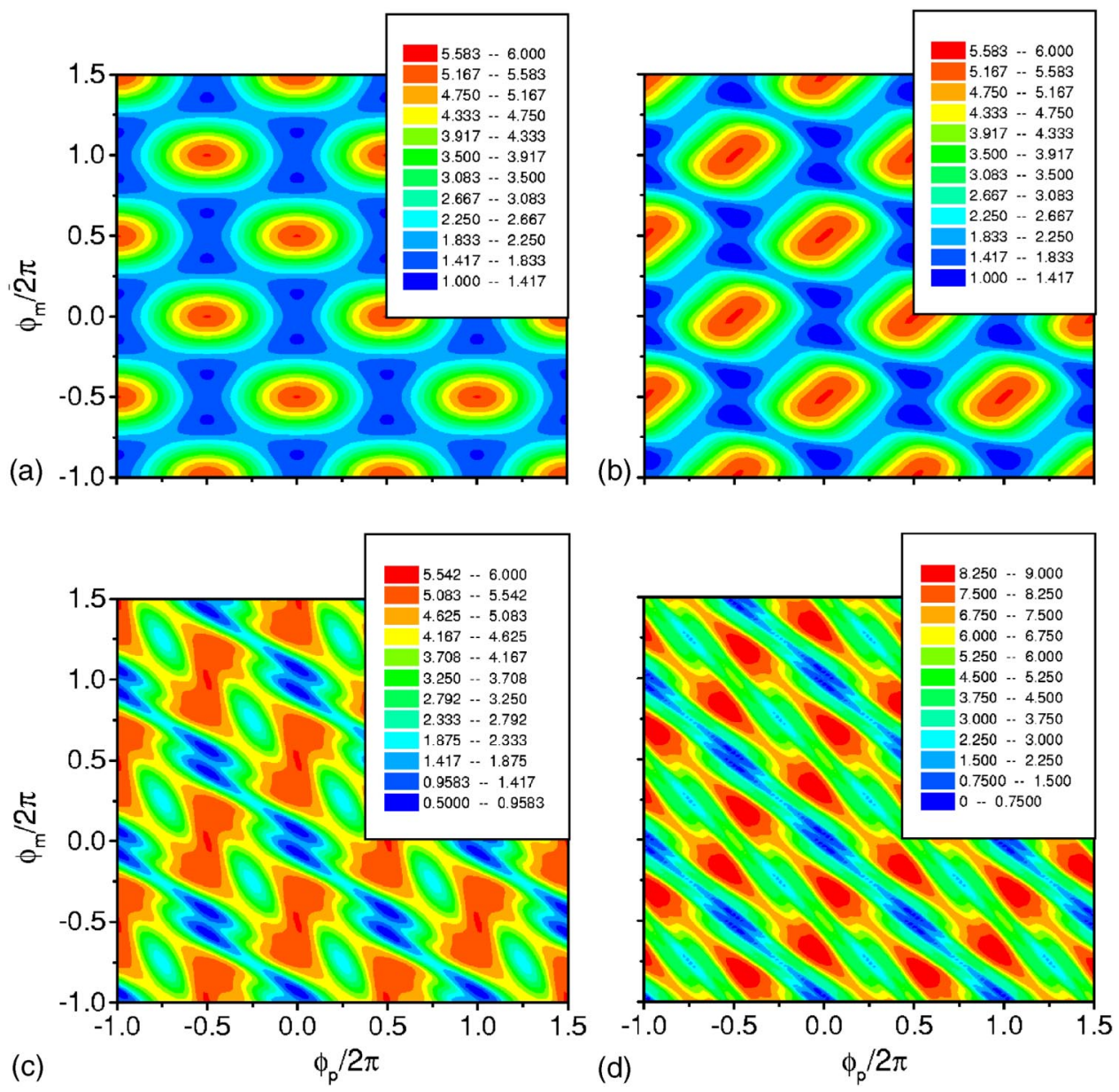

FIG. 2. (Color online) Contour plots of the potential energy $U\left(\phi_{p}, \phi_{m}\right)$, in units of $E_{J}$, for $\alpha$ $=0.8$ and $f=0.5$. Here $\beta_{L}$ $\equiv 2 \pi I_{0} L / \Phi_{0}=$ (a) 0 , (b) 1 , (c) 4 , and (d) 10. Notice that the welldefined double-well potential structure vanishes in (d), and thus the flux qubit breaks down. two adjoining double-well structures. These newly developed local minima will affect the two-level system achieved for the qubit. When the loop inductance increases even more to $\beta_{L} \approx 10$ [see Fig. $2(\mathrm{~d})$ ], the periodic potential is even more distorted. In this case, the well-defined double-well potential structure vanishes, and thus the flux qubit breaks down.

\section{B. Energy spectrum} by

The energy spectrum and the eigenstates are determined

$$
H \Psi\left(\phi_{p}, \phi_{m}\right)=E \Psi\left(\phi_{p}, \phi_{m}\right) .
$$

Figure 3 shows the dependence of the energy levels on the magnetic flux for $\beta_{L} \leqslant 1$. Here we choose $E_{J}=35 E_{c}$, where the charging energy $E_{c}$ is defined as $E_{c}=e^{2} / 2 C$. These parameters are close to those used in a recently fabricated fluxqubit device. $^{5}$

Around $f=0.5$, in sharp contrast with the higher energy levels, the energy difference

$$
\Delta=\varepsilon_{1}-\varepsilon_{0}
$$

between the lowest two levels is not sensitive to the variation of $\beta_{L}$. In Fig. 4, we show the energy separation of the two lowest levels $\Delta$ as a function of $\beta_{L}$. We find the interesting result that $\Delta\left(\beta_{L}\right)$ is almost flat at $f=0.5\left(0.011<\Delta\left(\beta_{L}\right) /\right.$ $E_{J}<0.0135$ ) when $0 \leqslant \beta_{L} \leqslant 0.85$ These features indicate that, even with a large loop inductance of $\beta_{L}=1$, in the vicinity of $f=0.5$ the two lowest eigenstates (denoted by $|0\rangle$ and $|1\rangle$ for the ground and the first excited states, respectively) remain suitable basis states for a flux qubit. Within the subspace of qubit states spanned by $|0\rangle$ and $|1\rangle$, the Hamiltonian is reduced to

$$
H=\varepsilon_{1}|1\rangle\left\langle 1\left|+\varepsilon_{0}\right| 0\right\rangle\langle 0| .
$$

If the average energy $\left(\varepsilon_{1}+\varepsilon_{0}\right) / 2$ is chosen to be the new zero-point energy of the flux qubit, then the Hamiltonian can be further expressed as

$$
H=\frac{1}{2} \Delta \rho_{z},
$$

where $\rho_{z}=|1\rangle\langle 1|-| 0\rangle\langle 0|$.

\section{Comparision with other works}

In Ref. 24, the effects of the loop inductance in a flux qubit are considered using a perturbation approach, where the Hamiltonian is expanded into three parts: an inductancefree Hamiltonian, an inductance-related harmonic oscillator term, and a small correction term. This perturbation method is valid for $\beta_{L} \ll 1$ because the correction term is proportional to the loop inductance of the flux qubit. Instead of using the perturbation approach, we numerically solve Eq. (11) to obtain the eigenvalues and eigenstates of the system. This numerical method allows us to extend our study to the regime 

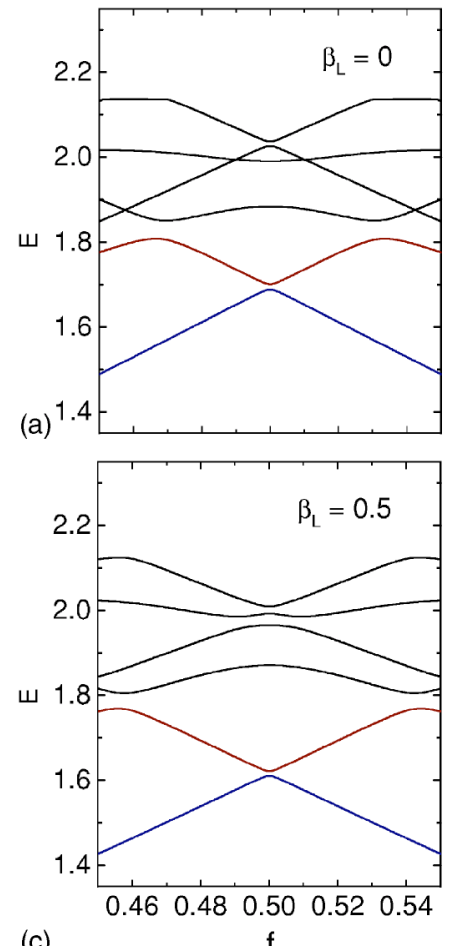

(c)

$f$
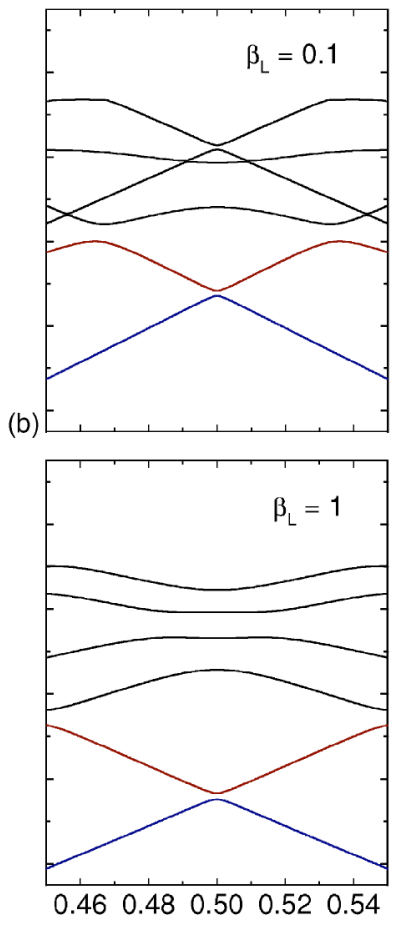

(d)

FIG. 3. (Color online) Energy levels of a single flux qubit vs reduced flux $f$ for different values of $\beta_{L}$, where only the levels of the states $|i\rangle, i=0$ to 5 , are shown. Here the energy $E$ is in units of $E_{J}$. Notice the robustness of the two lowest levels for wide changes in the loop inductance $L$.

of $\beta_{L} \sim 1$, where the lowest two eigenstates of the system can still be used for achieving a qubit. Using the experimental value $^{5} I_{0} \sim 0.5 \mu \mathrm{A}$, this regime corresponds to a loop inductance of $L \sim 1 \mathrm{nH}$.

\section{COUPLED FLUX QUBITS}

\section{A. The model}

To couple two flux qubits, we use a common inductance $L_{c}$ shared by these two qubits [see Fig. 1(b)]. Here the external flux $\Phi_{e}$ is applied within the loop $A_{1} L_{c} B_{1} A_{1}$. Also, the circuit is designed in such a way that the mutual inductance between loops $A_{1} L_{c} B_{1} A_{1}$ and $A_{1} B_{1} B_{2} A_{2} A_{1}$ may be ignored. This is achieved when only a small fraction of the flux generated by one loop passes through the other. (If this were not to be the case, the interbit coupling can still be achieved by the common inductance $L_{c}$, but the interaction Hamiltonian takes a more complicated form.) Phase drops through the three Josephson junctions of the $i$ th flux qubit are constrained by

$$
\phi_{1}^{(i)}-\phi_{2}^{(i)}+\phi_{3}^{(i)}+2 \pi\left[f+\left(I_{i} L_{i}+I_{j} L_{c}\right) / \Phi_{0}\right]=0,
$$

where $i, j=1,2(i \neq j)$, and

$$
\begin{gathered}
L_{1}=L_{c}+L_{b 1}, \\
L_{2}=L_{c}+L_{12}+L_{b 2} .
\end{gathered}
$$

The total supercurrent through $L_{c}$ is

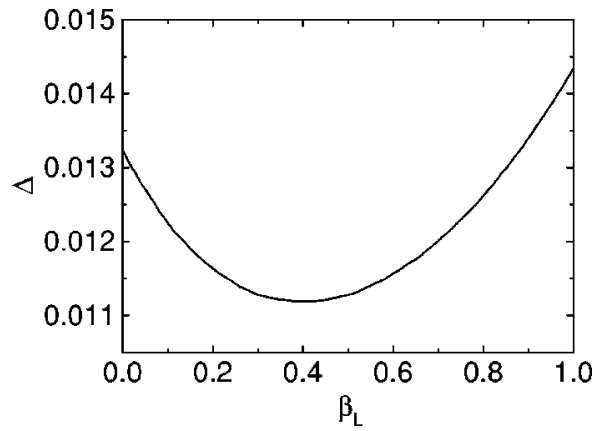

FIG. 4. Energy difference $\Delta$ between qubit states $|1\rangle$ and $|0\rangle$ as a function of $\beta_{L}$ for $f=0.5$. Here $\Delta$ is in units of $E_{J}$. Notice that the energy difference varies $\sim 0.003 E_{J}$ when varying the loop inductance $L$.

$$
I=I_{1}+I_{2}
$$

where

$$
I_{i}=I_{0 i} \sin \left(\phi_{p i}+\phi_{m i}\right),
$$

with $I_{0 i}=2 \pi E_{J}^{(i)} / \Phi_{0}$, and

$$
\begin{aligned}
& \phi_{p i}=\frac{1}{2}\left[\phi_{1}^{(i)}+\phi_{2}^{(i)}\right], \\
& \phi_{m i}=\frac{1}{2}\left[\phi_{1}^{(i)}-\phi_{2}^{(i)}\right] .
\end{aligned}
$$

The Hamiltonian of the two coupled flux qubits can be written as

$$
H=H_{1}+H_{2}+H_{I} \text {. }
$$

Here $H_{i}$ is the Hamiltonian of the $i$ th isolated flux qubit, with loop inductance $L_{i}$ and circulating supercurrent $I_{i}$, which has the form in Eq. (5) but with $f^{\prime}$ replaced by

$$
f_{i}^{\prime}=f+\frac{I_{i} L_{i}}{\Phi_{0}} .
$$

Also, $E_{J}, C$, and $\alpha$ are replaced by $E_{J}^{(i)}, C^{(i)}$ and $\alpha_{i}$. The interaction Hamiltonian is

$$
H_{I}=L_{c} I_{1} I_{2}-\sum_{i=1}^{2} \alpha_{i} E_{J}^{(i)} \Pi_{i},
$$

where

$$
\Pi_{i}=\cos \left(\gamma_{i}+2 \pi I_{j} L_{c} / \Phi_{0}\right)-\cos \gamma_{i}
$$

with

$$
\gamma_{i}=2 \pi f_{i}^{\prime}+2 \phi_{m i}
$$

and $i \neq j$.

When $\beta_{L i} \equiv 2 \pi I_{0 i} L_{c} / \Phi_{0} \ll 1, H_{I}$ is approximated by

$$
H_{I}=-L_{c} I_{1} I_{2}
$$

because

$$
\alpha_{i} E_{J}^{(i)} \Pi_{i} \approx L_{c} I_{1} I_{2}
$$

in this case (see the Appendix). Within the qubit-state subspace of the $i$ th isolated flux qubit, $H_{i}$ is reduced to 


$$
H_{i}=\frac{1}{2} \Delta_{i} \rho_{z}^{(i)},
$$

where

$$
\rho_{z}^{(i)}=\left|1_{i}\right\rangle\left\langle 1_{i}|-| 0_{i}\right\rangle\left\langle 0_{i}\right| .
$$

In the vicinity of $f=0.5$, because the supercurrents $I_{i}$ at states $\left|1_{i}\right\rangle$ and $\left|0_{i}\right\rangle$ have equal magnitudes but opposite directions, $I_{i}$ can be written as

$$
I_{i}=a_{i} \rho_{z}^{(i)}+b_{i}\left|1_{i}\right\rangle\left\langle 0_{i}\left|+b_{i}^{*}\right| 0_{i}\right\rangle\left\langle 1_{i}\right|,
$$

where

$$
\begin{aligned}
& a_{i}=\left\langle 1_{i}\left|I_{i}\right| 1_{i}\right\rangle, \\
& b_{i}=\left\langle 1_{i}\left|I_{i}\right| 0_{i}\right\rangle .
\end{aligned}
$$

Because the supercurrent $I_{i}$ at state $\left|1_{i}\right\rangle$ (i.e., $a_{i}$ ) is proportional to the slope of the energy level that corresponds to state $\left|1_{i}\right\rangle$ with respect to $f$ (see, e.g., Ref. 19), it falls to zero at the symmetric point $f=0.5$, where the level becomes flat. Also, our numerical results show that $b_{i}$ becomes a real number at $f=0.5$. Thus, we can rewrite $I_{i}$ at $f=0.5$ as

$$
I_{i}=b_{i} \rho_{x}^{(i)},
$$

with

$$
\rho_{x}^{(i)}=\left|1_{i}\right\rangle\left\langle 0_{i}|+| 0_{i}\right\rangle\left\langle 1_{i}\right| .
$$

For $\beta_{L i} \ll 1$, i.e., the common inductance $L_{c}$ is very small, the Hamiltonian at $f=0.5$ can be cast to

$$
H=\sum_{i=1}^{2} \frac{1}{2} \Delta_{i} \rho_{z}^{(i)}-\chi \rho_{x}^{(1)} \rho_{x}^{(2)},
$$

with

$$
\chi=L_{c} b_{1} b_{2} .
$$

It is clear that the interbit coupling persists at $f=0.5$.

\section{B. Energy spectrum}

Figure 5 shows the energy spectrum of the two coupled flux qubits around $f=0.5$. In order to realize fast two-bit operations while keeping the leakage from the qubit states to other higher-energy states small, we choose the interbit coupling strength to be comparable to the energy difference at $f=0.5$, between the basis states $\left|1_{i}\right\rangle$ and $\left|0_{i}\right\rangle$ of each qubit. As shown in Figs. 5(a) and 5(b), the energy spectrum remains similar in the vicinity of $f=0.5$ when the two flux qubits have different values of parameters. Furthermore, the two higher energy levels, $\epsilon_{3}$ and $\epsilon_{4}$, in the first four energy levels (i.e., $\boldsymbol{\epsilon}_{k}$ with $k=1$ to 4 ) of the two coupled flux qubits are flat in a relatively broad range around $f=0.5$; this flat region is much broader than the corresponding flat-energy-level range of the single flux qubit around $f=0.5$. The fluxindependent level $\epsilon_{3}$ in Fig. 5(a) corresponds to a singlet eigenstate, while other three levels correspond to triplet eigenstates. As expected, the transitions between this singlet state and other three triplet states are not allowed by the microwave perturbation [cf. Fig. 6(a)].
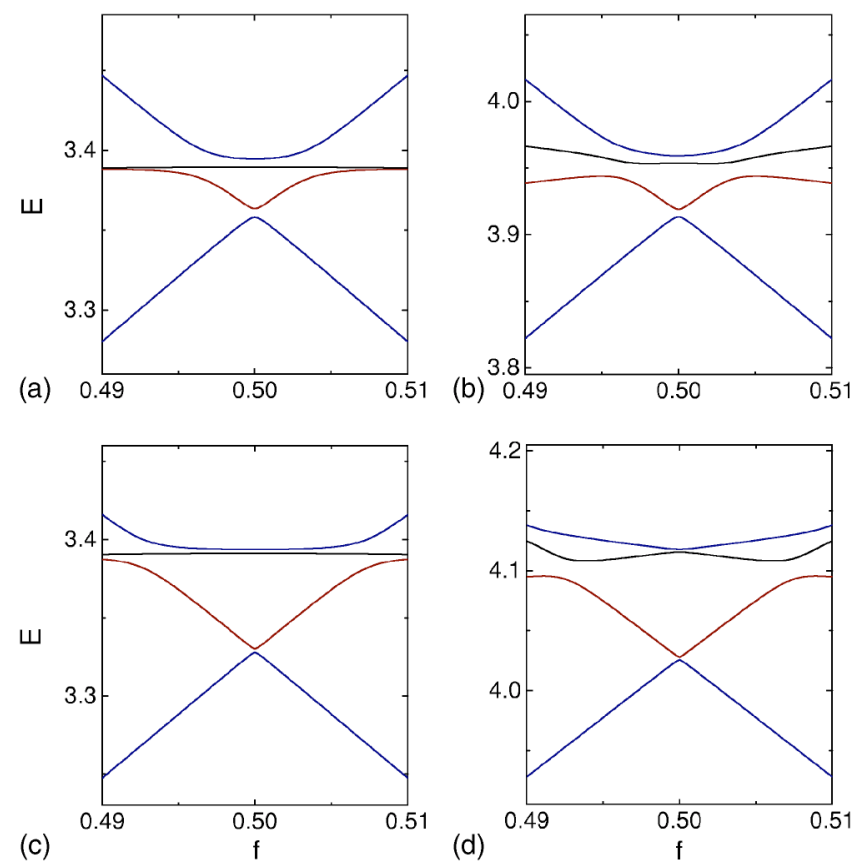

FIG. 5. (Color online) Energy levels of two coupled flux qubits vs reduced flux $f$ for $L_{b 1} / L_{c}=0.1$ and $\left(L_{12}+L_{b 2}\right) / L_{c}=0.2$. The parameters $\beta_{L i} \equiv 2 \pi I_{0 i} L_{c} / \Phi_{0}$ are (a) $\beta_{L 1}=\beta_{L 2}=0.03$; (b) $\beta_{L 1}=0.03$, $\beta_{L 2}=0.04$; (c) $\beta_{L 1}=\beta_{L 2}=0.07$; and (d) $\beta_{L 1}=0.07, \beta_{L 2}=0.1$. Here the energy $E$ is in units of $E_{J}^{(1)}$. Near $f=0.5$, the energy levels are robust with respect to the asymmetry between $\beta_{L 1}$ and $\beta_{L 2}$.

When the interbit coupling increases further, the flat region for both levels $\epsilon_{3}$ and $\epsilon_{4}$ widens for two qubits having identical parameters [see Fig. 5(c)], but $\epsilon_{3}$ and $\epsilon_{4}$ become much different in this region when the two qubits are not identical [see Fig. 5(d)]. Moreover, it can be seen that, at $f$ $=0.5$, the gap between levels $\epsilon_{1}$ and $\epsilon_{2}$ and that between $\epsilon_{3}$ and $\epsilon_{4}$ become narrow when increasing the interbit coupling.

At $f=0.5$, the first four energy levels, $\epsilon_{k}, k=1$ to 4 , of the coupled flux qubits can be approximated by

$$
\begin{aligned}
& \epsilon_{1}=-\frac{1}{2} E_{A}, \epsilon_{3}=\frac{1}{2} E_{B}, \\
& \epsilon_{2}=-\frac{1}{2} E_{B}, \epsilon_{4}=\frac{1}{2} E_{A},
\end{aligned}
$$

where

$$
\begin{aligned}
& E_{A}=\left[\left(\Delta_{1}+\Delta_{2}\right)^{2}+4 \chi^{2}\right]^{1 / 2}, \\
& E_{B}=\left[\left(\Delta_{1}-\Delta_{2}\right)^{2}+4 \chi^{2}\right]^{1 / 2} .
\end{aligned}
$$

The gap between levels $\epsilon_{2}$ and $\epsilon_{3}$ is $E_{B}$, which increases with $\chi$. The gap between levels $\epsilon_{1}$ and $\epsilon_{2}$ and that between $\epsilon_{3}$ and $\epsilon_{4}$ are given by $E_{A}-E_{B}$. Figures 5(a) and 5(b) correspond to $\chi \approx \Delta_{1}$; in Fig. 5(a) where $\Delta_{1}=\Delta_{2}=\Delta$, the two equal gaps, $E_{A}-E_{B}$, at $f=0.5$ are $(\sqrt{2}-1) \Delta$. When $\chi$ further increases, the value of $E_{A}-E_{B}$ decreases; namely, the two equal gaps become narrow [cf. Figs. 5(c) and 5(d)].

In the case of Fig. 5(a), because $2 \pi I_{0 i} L_{c} / \Phi_{0}=0.03$, the common inductance is 

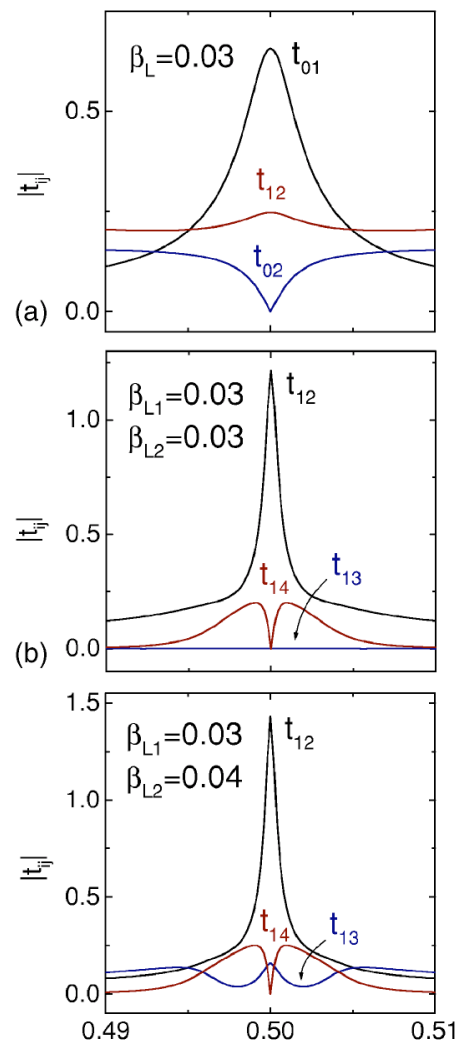

(c)

$\mathrm{f}$
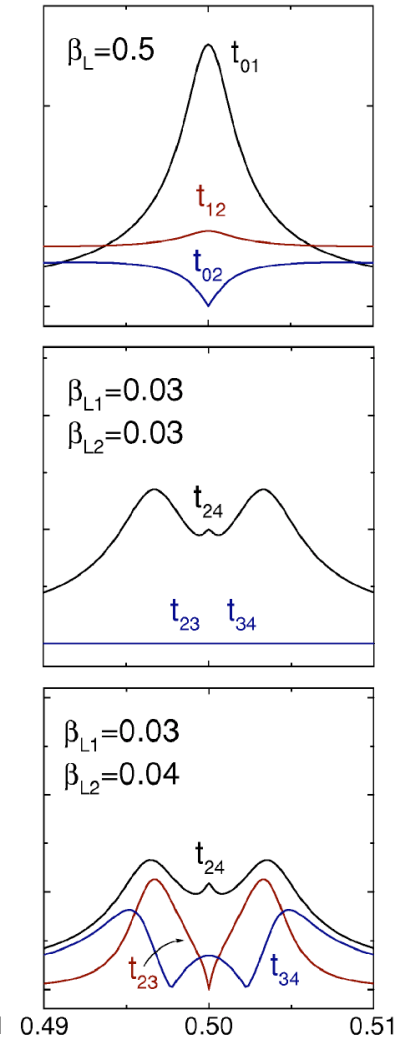

f

FIG. 6. (Color online) (a) Moduli of the transition matrix elements $t_{i j}$ between single-qubit states $|i\rangle$ and $|j\rangle$ vs reduced flux $f$. (b) and (c) Moduli of the transition matrix elements between coupledqubit states $\left|\epsilon_{i}\right\rangle$ and $\left|\epsilon_{j}\right\rangle$ vs $f$ for $L_{b 1} / L_{c}=0.1$ and $\left(L_{12}+L_{b 2}\right) / L_{c}$ $=0.2$. Here $\left|t_{i j}\right|$ is in units of $I_{0} \Phi_{X}$ in (a) and $I_{01} \Phi_{X}$ in (b) and (c). Note that, in (b) and (c), some transitions for the coupled two qubits are sensitive with respect to the asymmetry of $\beta_{L 1}$ and $\beta_{L 2}$.

$$
L_{c} \approx 20 \mathrm{pH}
$$

if the critical currents $I_{0 i}$ are equal to the experimental value ${ }^{5}$ $I_{0} \sim 0.5 \mu \mathrm{A}$. Such a small inductance is experimentally realizable, e.g., using a loop of diameter $d \approx 16 \mu \mathrm{m}$. Also, our numerical calculations show that $b_{i} \approx 0.66 I_{0}$ at $f=0.5$. The interbit coupling is thus of the order

$$
\chi=L_{c} b_{1} b_{2} \approx 0.013 E_{J},
$$

which is equal to the energy difference $\Delta$ at $f=0.5$ of the single flux qubit with $\beta_{L}=0.03$. The typical two-bit operation time related with the interbit-coupling strength is

$$
\tau_{2} \sim \hbar / \chi \text {. }
$$

Because $\chi \approx \Delta$ when $L_{c} \approx 20 \mathrm{pH}$,

$$
\tau_{2} \sim \tau_{1} \equiv \hbar / \Delta
$$

where $\tau_{1}$ is the one-bit operation time. Therefore, the corresponding two-bit operation is as fast as the one-bit operation.

\section{Comparison with other works}

Spectral results similar to the ones shown in Figs. 5(a) and 5(b) were also obtained by Storcz and Wilhelm ${ }^{21}$ and by

Majer et al. ${ }^{22}$ using simpler model Hamiltonians for two coupled flux qubits. However, because a different setup is used in Ref. 22 for coupling the two qubits, the four energy levels are flipped as compared to ours.

In our proposed setup, the shared part of the loop is on the same side of each qubit and, as given in Eq. (37), $\chi>0$. Since the negative coupling term in Eq. (33) favors the parallel arrangement of the (pseudo)spins, the interbit coupling here is ferromagnetic. In contrast, in the setup of Ref. 22, the shared loop is on the opposite side of each qubit. This gives rise to a negative coupling parameter $\chi$. Therefore, the interbit coupling there is antiferromagnetic because the (pseudo)spins tend to arrange antiparallel to each other.

Here we use the common loop inductance to couple two flux qubits, while different setups are proposed in Refs. 19 and 20, where the mutual inductance is used for coupling flux qubits. Since the mutual inductance is always smaller (sometimes can be much smaller) than the loop inductance of each flux qubit, if the two flux qubits are coupled via the mutual inductance (instead of the common loop inductance in our approach), a larger (sometimes much larger) loop is required for each qubit to produce a strong-enough interbit coupling. Therefore, due to the larger loop in each flux qubit, the system would experience more serious flux noise. This is a very significant difference between these approaches.

In our approach, we treat both single and coupled flux qubits using more realistic models, including the loop inductance. If our more general theory were used to deal with the different setup proposed in Ref. 22, for example, the simple model Hamiltonian used in Ref. 22 would be an approximation of the more general theory. The simpler model can explain the current experiment in Ref. 22, and so does the general theory. Moreover, the more general theory presented here can explain additional features that would be relevant for future experiments, while the simple model might not. For instance, even for the single flux qubit, the simple model cannot tell how large the loop should be to break down the qubit. Moreover, the simple model involves only two levels for each qubit. It cannot explain phenomena related to the state transitions from these two levels to higher ones. However, the general theory can do it.

\section{STATE TRANSITIONS}

\section{A. Transition matrix elements for single and coupled two qubits}

When a microwave field with an appropriate frequency $\omega$ is applied through the superconducting loop of the single flux qubit, a transition between two states occurs. Now, the total flux within the loop is $\Phi_{e}+\Phi_{f}$, where

$$
\Phi_{f}(t)=\Phi_{X} \cos (\omega t+\theta)
$$

is the microwave-field-induced flux through the loop. For a weak microwave field, the single flux qubit experiences a time-dependent perturbation

$$
H^{\prime}(t)=-I \Phi_{X} \cos (\omega t+\theta)
$$

and the transition matrix element $t_{i j}$ between states $|i\rangle$ and $|j\rangle$ is given by 


$$
t_{i j}=\left\langle i\left|I \Phi_{X}\right| j\right\rangle \text {. }
$$

Similarly, when the microwave field is applied through the left loop $A_{1} L_{c} B_{1} A_{1}$ of the coupled flux qubits, the transition matrix element $t_{i j}$ between the coupled-qubit states $\left|\epsilon_{i}\right\rangle$ and $\left|\epsilon_{j}\right\rangle$ is then

$$
t_{i j}=\left\langle\epsilon_{i}\left|I \Phi_{X}\right| \epsilon_{j}\right\rangle
$$

Note the difference between Eqs. (42) and (43), though they look similar to each other in expression; in Eq. (42), $|i\rangle$ is an eigenstate of the single flux qubit and $I$ is the circulating current in the qubit loop, while $I$ in Eq. (43) is the total current $I=I_{1}+I_{2}$ in the coupled-qubit circuit and $\left|\epsilon_{i}\right\rangle$ is an eigenstate of the coupled two flux qubits.

Figure 6(a) presents the flux dependence of $\left|t_{i j}\right|$ for transitions $|0\rangle \rightarrow|1\rangle,|0\rangle \rightarrow|2\rangle$, and $|1\rangle \rightarrow|2\rangle$ in a single flux qubit. Because of the symmetry of the wave functions, $\left|t_{02}\right|=0$ at $f=0.5$, and thus the transition $|0\rangle \rightarrow|2\rangle$ is forbidden. Also, it can be seen that $\left|t_{01}\right|$ is not sensitive to the variation of $\beta_{L}$, while $\left|t_{02}\right|$ and $\left|t_{12}\right|$ are slightly reduced when increasing $\beta_{L}$. This observation is consistent with the energy spectrum in Fig. 3, where the gap between the lowest two levels 0 and 1 is not significantly changed, but the gap between levels 1 and 2 slightly increases with $\beta_{L}$. In Figs. 6(b) and 6(c), we show the flux dependence of $\left|t_{i j}\right|$ for all possible transitions in the coupled flux qubits. When the two flux qubits have the same parameters, the transitions

$$
\begin{gathered}
\left|\epsilon_{1}\right\rangle \rightarrow\left|\epsilon_{3}\right\rangle, \\
\left|\epsilon_{2}\right\rangle \rightarrow\left|\epsilon_{3}\right\rangle, \\
\left|\epsilon_{3}\right\rangle \rightarrow\left|\epsilon_{4}\right\rangle
\end{gathered}
$$

are forbidden because $\left|t_{i j}\right|=0$ [see Fig. 6(b)]. However, they are allowed (except for some specific values of $f$ ) when the parameters of the two flux qubits are different [see Fig. 6(c)]. These properties are attributed to the changes in the symmetry of the qubit states. In particular, when $f=0.5,\left|t_{12}\right|$ has the largest value, while $\left|t_{24}\right|$ has a smaller value and others are either zero or much smaller.

\section{B. One- and two-bit operations implemented via microwave fields}

For a single flux qubit with $\beta_{L}=0.03$, the energy difference $\Delta$ between states $|1\rangle$ and $|0\rangle$ is $0.01291 E_{J}$ at $f=0.5$. Using an experimental value ${ }^{5}$ for the critical current $I_{0}$ $\sim 0.5 \mu \mathrm{A}$, we obtain $E_{J} \sim 1.03 \mathrm{meV}$. The energy difference of $0.01291 E_{J}$ corresponds to a gap of $\nu \approx 3.2 \mathrm{GHz}$. The onebit operation can be implemented using a resonant microwave field. For a weak driving field, the Rabi frequency $\Omega_{01}$ is given by $\left|t_{01}\right| / \hbar$. The typical switching time is $t_{\mathrm{SW}}$ $=\pi / \Omega_{01}$ when the states $|0\rangle$ and $|1\rangle$ flip. For instance, because $\left|t_{01}\right| \approx 0.66 I_{0} \Phi_{X}$ at $f=0.5$, the switching time $t_{\mathrm{SW}}$ is about $3 \mathrm{~ns}$ for $I_{0} \Phi_{X} \sim 1 \mu \mathrm{eV}$. If the leakage from these two states to others is small, one can realize a fast one-bit operation, e.g., with a switching time

$$
t_{\mathrm{SW}}=\frac{\pi}{\Omega_{01}} \sim 10 \nu^{-1}(\approx 3 \mathrm{~ns}),
$$

by increasing the microwave-field intensity.

Let the energy difference between states $|0\rangle(|1\rangle)$ and $|1\rangle$ $(|2\rangle)$ be $\hbar \omega_{01}\left(\hbar \omega_{12}\right)$. When the field is tuned to be resonant with the transition $|0\rangle \rightarrow|1\rangle$, the ratio of the transition probabilities between $|1\rangle \rightarrow|2\rangle$ and $|0\rangle \rightarrow|1\rangle$ can be estimated as

$$
\frac{\rho_{12}}{\rho_{01}}=\left(\frac{\Omega_{12}}{\Omega}\right)^{2} \frac{\sin ^{2}(\Omega \tau / 2)}{\sin ^{2}\left(\Omega_{01} \tau / 2\right)},
$$

where

$$
\Omega=\left[\Omega_{12}^{2}+\left(\omega_{12}-\omega_{01}\right)^{2}\right]^{1 / 2},
$$

$\Omega_{12}=\left|t_{12}\right| / \hbar$, and $\tau$ is the duration of the microwave-field pulse. When

$$
\tau=\pi / \Omega_{01} \sim 10 \nu^{-1},
$$

using the numerical results $\hbar \omega_{01}=0.01291 E_{J}, \quad \hbar \omega_{12}$ $=0.18763 E_{J}$, and $\left|t_{12} / t_{01}\right| \approx 0.38$ at $f=0.5$, we have

$$
\frac{\rho_{12}}{\rho_{01}} \approx 1.5 \times 10^{-6} \text {. }
$$

This implies that the leakage to other states is small for a fast one-bit operation implemented via a microwave field.

Corresponding to Fig. 5(a), $\left|\epsilon_{1}\right\rangle$ and $\left|\epsilon_{2}\right\rangle$ at $f=0.5$ are approximated by

$$
\begin{gathered}
\left|\epsilon_{1}\right\rangle=\frac{1}{\sqrt{\eta^{2}+1}}(\eta|00\rangle+|11\rangle), \\
\left|\epsilon_{2}\right\rangle=\frac{1}{\sqrt{2}}(|01\rangle+|10\rangle),
\end{gathered}
$$

with

$$
\eta=\frac{\Delta+\left(\Delta^{2}+\chi^{2}\right)^{1 / 2}}{\chi} .
$$

Initially preparing the system at the (entangled) ground state $\left|\epsilon_{1}\right\rangle$, one can produce the maximally entangled state $\left|\epsilon_{2}\right\rangle$ using a microwave-field pulse of duration $\tau=\pi / \Omega_{12}$, where the Rabi frequency $\Omega_{12}$ is given by $\left|t_{12}\right| / \hbar$ for a weak driving field.

At $f=0.5$, we have $\hbar \omega_{12}=0.00528 E_{J}, \hbar \omega_{24}=0.03124 E_{J}$, and $\left|t_{24} / t_{12}\right| \approx 0.41$. When the microwave field is in resonance with the transition $\left|\epsilon_{1}\right\rangle \rightarrow\left|\epsilon_{2}\right\rangle$ at $f=0.5$,

$$
\frac{\rho_{24}}{\rho_{12}} \approx 4.4 \times 10^{-6}
$$

for

$$
\tau=\pi / \Omega_{12} \sim 20 \pi / \omega_{12} .
$$

Because the state leakage is very small, a fast two-bit operation can also be implemented using a microwave field.

\section{SUPERCURRENTS AND QUANTUM MEASUREMENT}

The circulating supercurrents flowing through the inductance $L$ or $L_{c}$ are different for different eigenstates. This 

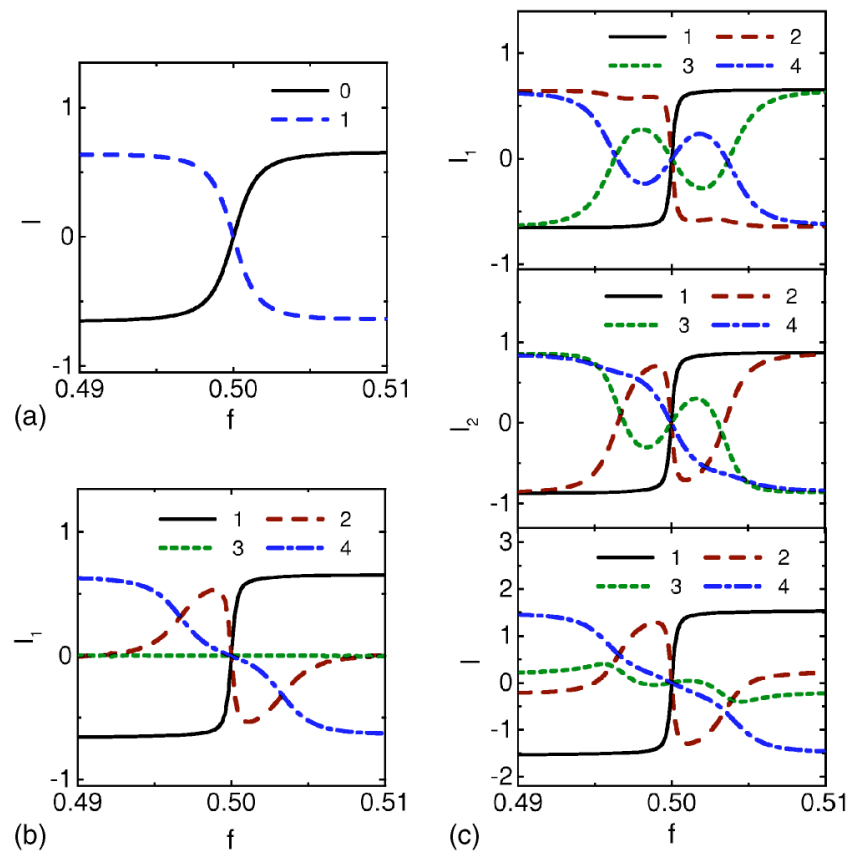

(b)

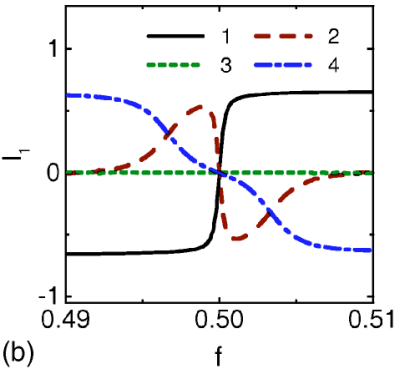

FIG. 7. (Color online) (a) Supercurrents $I$ vs reduced flux $f$ at eigenstates $|0\rangle$ and $|1\rangle$ of a single flux qubit for $\beta_{L}=0.03$. (b) Supercurrents $I_{1}$ vs $f$ at eigenstates $\left|\epsilon_{k}\right\rangle, k=1$ to 4 , of two coupled flux qubits for the symmetric circuit with $\beta_{L 1}=\beta_{L 2}=0.03$. (c) Supercurrents $I_{1}, I_{2}$, and $I$ vs $f$ at eigenstates $\left|\epsilon_{k}\right\rangle, k=1$ to 4 , of two coupled flux qubits for an asymmetric circuit with $\beta_{L 1}=0.03$ and $\beta_{L 2}=0.04$. Here we choose $L_{b 1} / L_{c}=0.1$ and $\left(L_{12}+L_{b 2}\right) / L_{c}=0.2$ for the coupled flux qubits. The supercurrents are in units of $I_{0}$ in (a) and $I_{01}$ in (b) and (c). For the coupled two qubits, the total current $I=I_{1}+I_{2}$ is robust with respect to the asymmetry of $\beta_{L 1}$ and $\beta_{L 2}$.

property can be used for implementing a readout of the qubit states. For a single flux qubit, around $f=0.5$, the supercurrents $I$ at eigenstates $|0\rangle$ and $|1\rangle$ (i.e., $\langle 0|I| 0\rangle$ and $\langle 1|I| 1\rangle$ ) have equal magnitudes but opposite directions [see Fig. 7(a)]. During quantum measurement, one can switch on the flux transformer to couple the inductance $L$ with a dc-SQUID magnetometer [cf. Fig. 1(a)] to distinguish the two eigenstates of the qubit because at these two states the supercurrents $I$ through $L$ generate two different fluxes in the SQUID loop of the magnetometer. In general, if the single flux qubit is at the superposition state $c_{1}|1\rangle+c_{0}|0\rangle$, the measurement will show that the qubit has probability $\left|c_{i}\right|^{2}$ at the eigenstate $|i\rangle$, where $i=0,1$. For the two coupled flux qubits, the supercurrents through the common inductance $L_{c}$ take different values at its four eigenstates.

Similar to the single flux qubit, a switchable flux transformer can be used to couple $L_{c}$ and the SQUID loop of the magnetometer for reading out the coupled-qubit states because the supercurrents $I$ at different eigenstates contribute different fluxes in the SQUID loop of the magnetometer. The supercurrents $I_{1}$ at the four eigenstates of the coupled qubits are shown in Fig. 7(b) for two flux qubits having identical parameters. Since $I_{1}=I_{2}$ in this case, the total supercurrent $I$ is $2 I_{1}$. When the parameters of the two flux qubits become different, the total supercurrents I look similar to those in Fig. 7(b), but $I_{1}$ and $I_{2}$ (which flow through the Josephson junctions of the qubits) change drastically [cf. Fig. 7(c)].
Also, it can be seen that at the eigenstates of the system the circulating supercurrents in both single and coupled flux qubits fall to zero at $f=0.5$. To read out the qubit states, one can shift the system away from this point.

\section{DISCUSSION AND CONCLUSION}

For the charge qubits coupled by $L C$-oscillator modes ${ }^{13}$ or by an inductance, ${ }^{14}$ the inductances proposed to be used are $\sim 3.6 \mu \mathrm{H}$ or $\sim 30 \mathrm{nH}$, respectively, for a two-bit operation ten times slower than the typical one-bit operation. An inductance for coupling charge qubits similar to that in Ref. 13, particularly, has a value much larger than $L_{c}(\approx 20 \mathrm{pH})$ for coupling flux qubits. It is difficult to fabricate in a small size without introducing a strong coupling with the environment. Because two-bit operations are much slower than one-bit operations in the inductively coupled charge qubits, an efficient scheme is thus required to minimize the number of two-bit (as opposed to one-bit) operations to obtain a conditional gate. ${ }^{14}$ However, for inductively coupled flux qubits, the above limitation in using two-bit operations for constructing a conditional gate is removed because two-bit operations can be as fast as one-bit operations. In this case, any schemes for constructing conditional gates become efficient by minimizing the number of operations that are used (either one- or two-bit). Note that the common inductance of $L_{c} \approx 20 \mathrm{pH}$ can produce a strong interbit coupling. As a result, two-bit as fast as one-bit operations can be achieved. This common inductance is comparable to the loop inductance, $L \sim 10 \mathrm{pH}$, of the single flux qubit currently realized in experiments.

To couple several flux qubits, the inductances of all loops involved could be small, comparable to the loop inductance of a single flux qubit currently realized in experiments. This is the case we studied in the present paper, where two coupled flux qubits are considered. If a number of flux qubits are coupled, then the inductances of some loops will become larger, but the common or shared inductance for producing the interbit coupling can still be chosen small (about $20 \mathrm{pH}$ ). If the circuits except for the line $A_{1} L_{c} B_{1}$ (corresponding to the common or shared inductance) could be screened from the environment (that is a big challenge for experimentalists for sure), the main noise would be due to the small common or shared inductance.

In conclusion, we have proposed an experimentally realizable method for inductively coupled flux qubits that can achieve two-bit operations performing as fast as one-bit operations. We treat both single and coupled flux qubits with more realistic models, including the loop inductance. Moreover, we show that the coupled flux qubits have different flux-dependent behaviors in the transitions between states. We find that the forbidden transitions in the coupled two flux qubits become allowed (except for some specific values of the external flux) when the parameters of the two qubits change from being initially equal to each other and then making these different.

\section{ACKNOWLEDGMENTS}

We thank J.S. Tsai, T. Yamamoto, Yu. Pashkin, O. Astafiev, Y.X. Liu, L.F. Wei, T. Tilma, F. Wilhelm, Q. Niu, and 
S.C. Bernstein for discussions and comments. This work was supported in part by the National Security Agency (NSA) and Advanced Research and Development Activity (ARDA) under Air Force Office of Research (AFOSR) Contract No. F49620-02-1-0334, and by the National Science Foundation Grant No. EIA-0130383. J.Q.Y. was also supported by the National Natural Science Foundation of China, Grant Nos. 10174075 and 10474013, and the Special Funds for Major State Basic Research of China Grant No. G2001CB3095.

\section{APPENDIX: SERIES EXPANSION OF THE INTERACTION HAMILTONIAN}

The supercurrent $I_{i}$ flows through each of the three Josephson junctions in the $i$ th flux qubit, so $I_{i}$ can also be written as

$$
I_{i}=\alpha_{i} I_{0 i} \sin \phi_{3}^{(i)}=-\alpha_{i} I_{0 i} \sin \left[2 \pi\left(f_{i}^{\prime}+I_{j} L_{c} / \Phi_{0}\right)+2 \phi_{m i}\right],
$$

where $i, j=1,2$ and $i \neq j$. Taking advantage of this relation for $I_{i}$, one can expand the interaction Hamiltonian (22) as

$$
H_{I}=-\lambda L_{c} I_{1} I_{2}-\sum_{i=1}^{2} \alpha_{i} E_{J}^{(i)} \xi_{i},
$$

where

$$
\lambda=1+\sum_{i=1}^{2}\left[\frac{1}{3} \beta_{L i}^{2}\left(\frac{I_{i}}{I_{0 i}}\right)^{2}+\frac{2}{15} \beta_{L i}^{4}\left(\frac{I_{i}}{I_{0 i}}\right)^{4}+\ldots\right],
$$

and

$$
\begin{aligned}
\xi_{i}= & \frac{1}{2} \cos \left(2 \pi f_{i}^{\prime}+2 \phi_{m i}\right) \\
& \times\left[\beta_{L j}^{2}\left(\frac{I_{j}}{I_{0 j}}\right)^{2}+\frac{5}{12} \beta_{L j}^{4}\left(\frac{I_{j}}{I_{0 j}}\right)^{4}+\ldots\right],
\end{aligned}
$$

with

$$
\beta_{L i} \equiv 2 \pi I_{0 i} L_{c} / \Phi_{0}<\pi / 2 .
$$

The term $-\lambda L_{c} I_{1} I_{2}$ in $H_{I}$ produces an interbit coupling between flux qubits 1 and 2, while $\alpha_{i} E_{J}^{(i)} \xi_{i}$ slightly modifies the energy levels of the $i$ th flux qubit.
*Email address: jqyou@fudan.edu.cn

${ }^{\dagger}$ Email address: yasunobu@frl.cl.nec.co.jp

*Email address: nori@umich.edu

${ }^{1}$ Y. Nakamura, Yu. A. Pashkin, and J. S. Tsai, Nature (London) 398, 786 (1999).

${ }^{2}$ D. Vion, A. Aassime, A. Cottet, P. Joyez, H. Pothier, C. Urbina, D. Esteve, and M. H. Devoret, Science 296, 886 (2002).

${ }^{3}$ J. R. Friedman, V. Patel, W. Chen, S. K. Tolpygo, and J. E. Lukens, Nature (London) 406, 43 (2000).

${ }^{4}$ J. E. Mooij, T. P. Orlando, L. Levitov, L. Tian, C. H. van der Wal, and S. Lloyd, Science 285, 1036 (1999); C. H. van der Wal, A. C.J. ter Haar, F. K. Wilhelm, R. N. Schouten, C. J.P.M. Harmans, T. P. Orlando, S. Lloyd, and J. E. Mooij, ibid. 290, 773 (2000).

${ }^{5}$ I. Chiorescu, Y. Nakamura, C. J.P.M. Harmans, and J. E. Mooij, Science 299, 1869 (2003).

${ }^{6}$ Y. Yu, S. Y. Han, X. Chu, S. I. Chu, and Z. Wang, Science 296, 889 (2002); J. M. Martinis, S. Nam, J. Aumentado, and C. Urbina, Phys. Rev. Lett. 89, 117901 (2002).

${ }^{7}$ Yu. A. Pashkin, T. Yamamoto, O. Astafiev, Y. Nakamura, D. V. Averin, and J. S. Tsai, Nature (London) 421, 823 (2003); T. Yamamoto, Yu. A. Pashkin, O. Astafiev, Y. Nakamura, and J. S. Tsai, ibid. 425, 941 (2003).

${ }^{8}$ A. J. Berkley, H. Xu, R. C. Ramos, M. A. Gubrud, F. W. Strauch, P. R. Johnson, J. R. Anderson, A. J. Dragt, C. J. Lobb, and F. C. Wellstood, Science 300, 1548 (2003).

${ }^{9}$ D. V. Averin and C. Bruder, Phys. Rev. Lett. 91, 057003 (2003).

${ }^{10}$ A. Blais, A. Maassen van den Brink, and A. M. Zagoskin, Phys. Rev. Lett. 90, 127901 (2003).

${ }^{11}$ J. Siewert and R. Fazio, Phys. Rev. Lett. 87, 257905 (2001).

${ }^{12}$ W. M. Kaminsky and S. Lloyd, quant-ph/0211152 (unpublished).
${ }^{13}$ Yu. Makhlin, G. Schön, and A. Shnirman, Nature (London) 398, 305 (1999).

${ }^{14}$ J. Q. You, J. S. Tsai, and F. Nori, Phys. Rev. Lett. 89, 197902 (2002). A longer version of it is available in cond-mat/0306203; see also New Directions in Mesoscopic Physics, edited by R. Fazio, V. F. Gantmakher, and Y. Imry (Kluwer, Dordrecht, 2003), p. 351.

${ }^{15}$ J. Q. You, J. S. Tsai, and F. Nori, Phys. Rev. B 68, 024510 (2003).

${ }^{16}$ J. Lantz, M. Wallquist, V. S. Shumeiko, and G. Wendin, Phys. Rev. B 70, 140507(R) (2004).

${ }^{17}$ Y. D. Wang, P. Zhang, D. L. Zhou, and C. P. Sun, Phys. Rev. B 70, 224515 (2004).

${ }^{18}$ C. H. van der Wal, F. K. Wilhelm, C. J.P.M. Harmans, and J. E. Mooij, Eur. Phys. J. B 31, 111 (2003); L. Tian, S. Lloyd, and T. P. Orlando, Phys. Rev. B 65, 144516 (2002).

${ }^{19}$ T. P. Orlando, J. E. Mooij, L. Tian, C. H. van der Wal, L. S. Levitov, S. Lloyd, and J. J. Mazo, Phys. Rev. B 60, 15398 (1999).

${ }^{20}$ M. J. Storcz and F. K. Wilhelm, Appl. Phys. Lett. 83, 2387 (2003).

${ }^{21}$ M. J. Storcz and F. K. Wilhelm, Phys. Rev. A 67, 042319 (2003).

${ }^{22}$ J. B. Majer, F. G. Paauw, A. C.J. ter Haar, C. J.P.M. Harmans, and J. E. Mooij, cond-mat/0308192 (unpublished); J. B. Majer, Ph.D. thesis, TU Delft, 2002.

${ }^{23}$ A. Izmalkov, M. Grajcar, E. Il'ichev, Th. Wagner, H.-G. Meyer, A. Yu. Smirnov, M. H.S. Amin, A. Maassen van den Brink, A. M. Zagoskin, Phys. Rev. Lett. 93, 037003 (2004).

${ }^{24}$ D. S. Crankshaw and T. P. Orlando, IEEE Trans. Appl. Supercond. 11, 1006 (2001). 